\title{
Further research is required to provide evidence of the effectiveness and feasibility of the nurse-led, case management approach to the care of older adults with chronic comorbid conditions
}

\section{Marianne Wallis}

Commentary on: Lupari M, Coates V, Adamson G, et al. 'We're just not getting it right' - how should we

School of Nursing and Midwifery, Griffith University, Gold Coast, Queensland, Australia

\section{Correspondence to:}

\section{Marianne Wallis}

School of Nursing and Midwifery, Griffith University, G16_2.69

Gold Coast Campus, Gold Coast, QLD 4222, Australia;

m.wallis@griffith.edu.au

provide care to the older person with multi-morbid chronic conditions? J Clin Nurs 2011;20:1225-35.

\section{Implications for practice}

- There is evidence that a nurse-led, case management approach is acceptable to patients with chronic comorbid conditions.

- There is no evidence from robust clinical trials that a nurse-led, case management approach results in more effective or more cost effective care.

\section{Implications for research}

More robust studies, focusing on the effectiveness, cost-effectiveness and acceptability of different models of health service delivery, including nurse-led models, need to be undertaken to inform future health service provision for the growing number of older adults with chronic, comorbid conditions.

\section{Context}

The global population is ageing as the number of older persons increases and birth rates decline. A consequence of this ageing of the population is an increased prevalence of chronic conditions and, in particular, multi-morbidity (ie, more than one coexisting chronic condition). The WHO estimates that chronic disease accounts for $60 \%$ of deaths worldwide and has given precedence globally to the prevention and treatment of chronic disease. ${ }^{1}$ Although some randomised controlled trials of nurse-led communitybased interventions, for specific conditions, have reported 
a positive effect on hospital re-admission rates, ${ }^{2}$ evidence of the effectiveness of nurse-led case management for older people with multi-morbid chronic conditions is lacking. ${ }^{3}$

\section{Methods}

The review by Lupari and colleagues was based on a systematic search that covered clinical trials registries to sources of grey literature. It is possible that some relevant studies were omitted as the search strategy was limited to English language articles. Methodologically, this review was hampered by the authors' broad interpretation of the term effectiveness. Effectiveness has not been differentiated from feasibility or acceptability. Studies with quite disparate research questions and methodologies were combined in this review. Studies were selected if they compared case management with usual care at home; however, the methods used to assess study quality and extract data were not described in sufficient detail. of 568 articles identified by the search strategy, 8 were considered appropriate to include in the review. Narrative and qualitative analyses were then undertaken on these articles.

\section{Findings}

The review found no evidence of a reduction in emergency admissions, length of hospital stay or costs associated with access to nurse-led, case management services for older adults with multi-morbid chronic conditions. The data from the qualitative studies and some service evaluation studies suggested that the case management approach is acceptable to clients, carers and health professionals, and it is a feasible model of service delivery with professional benefits for nurses and doctors. The data from this review, however, are insufficient for forming recommendations.

\section{Commentary}

Lupari and her colleagues undertook a review of research and service evaluation studies that they variously described as a literature review and a systematic review of the literature. Although the Cochrane Collaboration has promulgated the utility of the systematic review with meta-analysis as a tool for assessing the clinical effectiveness of single interventions, this approach has been less useful in assessing effectiveness of models of service delivery or bundled interventions. Thus, other approaches to synthesising the results of numerous disparate studies have been developed. More specifically, differentiations have been made between integrative and interpretive reviews or syntheses. ${ }^{4}$ In this context, integration involves aggregating quantitative data and the use of statistical meta-analysis, whereas interpretation involves creating meaning from multiple sources of qualitative and/or quantitative data. It appears that, quite properly, the authors undertook an interpretive synthesis of available qualitative and quantitative research studies. There were, however, a number of anomalies in the review. For example, appropriately, the authors did not report on a meta-analysis of the aggregate data from the quantitative studies, as the research designs and outcome measures were too dissimilar. This suggests that two studies were included in a qualitative synthesis, whereas six studies were included in a "quantitative synthesis (metaanalysis)'. In addition, the authors stated that the numbers of participants in the studies ranged from 19 to 597, but Study 2 - a cluster randomised controlled study - had 904 participants.

This review highlights the issue that numerous small studies are being undertaken at the local level. These studies appropriate the time, energy and funds of all involved yet do not provide robust evidence of the effectiveness of different models of service delivery in community settings. Before health systems are overwhelmed with the demand from people with multi-morbid chronic conditions, it would seem prudent that evidence-based systems are in place. Consequently, a suitable focus for the expenditure of large-scale research funds would be well-powered trials comparing the relative effectiveness of different models of service delivery.

\section{Competing interests None.}

\section{References}

1. World Health Organization. Preventing Chronic Disease: A Vital Investment. Geneva: WHO Global Report, 2005.

2. Taylor SJC, Bestall JC, Cotter S, et al. Clinical service organisation for heart failure. Cochrane Database Syst Rev 2005;2:CD002752.

3. Hutt R, Rosen R, McCauley J. Case-Managing Long-Term Conditions. What Impact Does It Have in the Treatment of Older People? London: King's Fund 2004.

4. Fleming K. EBN notebook. The synthesis of qualitative research and evidence-based nursing. EBN 2007;10:68-71.

5. Dixon-Woods M, Agarwal S, Jones D, et al. Synthesising qualitative and quantitative evidence: a review of possible methods. J Health Serv Res Policy 2005;10:45-53. 